\title{
Modern therapeutic aspects in two cases of congenital hyperinsulinism
}

\author{
Cristian Minulescu ${ }^{1,2}$, Dana Spirea ${ }^{3,4}$ \\ ${ }^{1}$ Dr. Cristian Minulescu PFA, MedLife, Bucharest, Romania \\ ${ }^{2}$ Société Francaise pour l'Etude des Maladies Héréditaires du Métabolisme, France \\ ${ }^{3}$ Clinical Children's Hospital, Brasov, Romania \\ ${ }^{4}$ The European Society for Paediatric Gastroenterology Hepatology and Nutrition
}

\begin{abstract}
In this article we will present the therapeutic and clinical aspects found in the case of two pediatric patients diagnosed with congenital hyperinsulinism. Thus, we want to show the modern therapeutic principles used in the treatment of these patients. It is important to remember that congenital hyperinsulinism is the only curable metabolic congenital disease, as currently surgical therapy is only indicated in isolated cases.

This is why quick diagnosis is essential, because the cases of hypoglycemia caused by this illness are severe. Furthermore, congenital hyperinsulinism blocks ketogenesis, so neither the brain nor the myocardium can use ketone bodies as an alternative energy substrate in case of hypoglycemia, and the risk of brain damage or cardiac arrest is very high in this disease.
\end{abstract}

Keywords: hypoketotic hypoglycemia, curable disease, diazoxide

\section{INTRODUCTION}

The pancreas is an organ with both exocrine and endocrine role. The most important hormone it secretes is insulin. Its roles are manifold, but the most important is decreasing glycemia (1). However, this glucose buffering capacity is limited, as the pancreas can only secrete enough insulin to buffer at most 10 $\mathrm{mg} / \mathrm{kg} / \mathrm{min}$ glucose; a higher quantity of glucose will overwhelm its capacity to secrete insulin and will cause hyperglycemia.

Another role of insulin is that of an anabolizer, favoring protein synthesis (2). This role is extremely important in patients with congenital metabolic diseases, both for the therapy of such diseases and to understand their physiopathology.

A decrease in insulin production leads to diabetes mellitus, and an increase in this production leads to a disorder called congenital hyperinsulinism. It has three main forms: one focal, one diffuse, and one called syndromic (3).

The most frequently encountered form is the focal one, in which there is an isolated hyperproliferation of pancreatic cells, leading to the appearance of an insulinoma.
In its diffuse form, this hyperproliferation includes the entire pancreas, and in the syndromic form hyperinsulinism appears in the context of a genetic syndrome, which has other manifestations in addition to the pancreatic anomaly (4).

\section{METHOD}

In this paper we will present the cases of two patients diagnosed and treated by the authors for congenital hyperinsulinism. The diagnosis was established by calculating the glucose flow needed to control hypoglycemia and the glucagon test. The role of genetic testing remains small in this pathology, but gene tests were performed on both patients.

\section{CASES PRESENTATION}

\section{Case 1}

This is a 5-month female infant, born of high degree consanguineous parents. Starting with the age of 2-3 days, she exhibited an altered general state and fine trembling of the lower limb extremities, in the context of $27 \mathrm{mg} / \mathrm{dl}$ glycemia. IV glucose therapy is 
started with $6 \mathrm{mg} / \mathrm{kg} / \mathrm{min}$, gradually increased to 8 $\mathrm{mg} / \mathrm{kg} / \mathrm{min}$. This is interpreted as neonatal sepsis and empirical antibiotherapy is started while monitoring glycemia. Under this treatment, she exhibits generalized tonic clonic seizures in the presence of $4 \mathrm{mg} / \mathrm{dl}$ glycemia.

At the Brasov hospital, the patient is hospitalized for 3 days, during which period she did not repeat the neurological manifestations, but the general state remained mediocre, with glycemia between 24 and 50 $\mathrm{mg} / \mathrm{dl}$ requiring continuous glucose intake of 9-11 $\mathrm{mg} / \mathrm{kg} / \mathrm{min}$. Transitory hypoglycemia associated with gestational diabetes and neonatal sepsis were ruled out.

During this hospitalization, laboratory investigations were continued, while reevaluating the septic profile, which revealed Klebsiella pn. in the umbilical secretion, and treatment was started according to the antibiogram. Hypoglycemia persists despite treatment, for which reason nutrition is supplemented with pulvis glucose in each baby bottle and repeated boluses of $10 \%$ glucose. Since the other causes for the newborn's severe hypoglycemia were ruled out, an endocrinological disturbance is considered, and for this reason ACTH, serum cortisol, STH, thyroid hormones, insulin and $\mathrm{C}$ peptide are sampled, all with normal values. Exploratory imaging (abdominal and transfontanellar ultrasound) was also normal. Serological investigations reveal hyperammonemia and lactic acid over the normal values, associated to a discrete hepatic cytolysis syndrome. Considering these changes, a suspicion appears about a glycogen storage disorder, as hepatomegaly may be absent during the neonatal age. Genetic samples are taken to confirm this diagnosis and the patient is released with recommendations to supplement oral glucose intake for each bottle of Polycal (maltodextrin) and Kreon at each meal, and to correct possible hypoglycemia $10 \%$ will be administered orally.

The genetic panel testing the 23 known genes that can cause glycogenosis is negative for each individual gene. Hypoglycemia persisted, and to ensure normal glycemia $10 \mathrm{mg} / \mathrm{kg} / \mathrm{min}$ glucose was used. At this moment, the suspicion appeared of congenital hyperinsulinism and a glucagon test was performed, which was positive. An initial treatment with octreotide was started. This led to an improvement in glycemia, but not normalization. This is why it was necessary that the milk received by the patient be enriched not only with dextrin maltose but also with glucose, in order to ensure increased glucose intake. As the baby was not feeding properly, gavage was used initially and then became discontinuous. Later a nutrition pump and a gastrostomy button were used. The patient was released with this treatment. Genetic testing was carried out using a panel including all genes that are known at this time to be involve in the pathogeny of hyperinsulinism, which were sequenced using the NGS technique. However, the result was negative.

A few months later, maintaining correct glycemia became increasingly difficult, and it was necessary to increase the flow of glucose even more. Under these circumstances, octreotide was replaced with diazoxide, which led to the glycemic profile quickly becoming normalized. Oral nutrition was resumed shortly, which allowed the gastrostomy to be removed. Rare hypoglycemia was found during the next months but later disappeared.

\section{Case 2}

This is a three-year-old girl that had several episodes of hypoglycemia. The patient was born prematurely at 34 weeks, and postnatal adaptation was difficult. Hypoglycemia appeared during the neonatal period, requiring glucose therapy with $16 \mathrm{mg} / \mathrm{kg} / \mathrm{min}$ flow to control it. No other investigations were carried out to establish its etiology. During the first year of life hypoglycemia persists, leading to generalized tonic clonic seizures. At the age of 9 months she was admitted for convulsions with hypoglycemic background, and the hospitalizations repeated every two months. At the age of two years, the ultrasound detects the left kidney larger than the right one, a hypertrophy of the left half of the tongue and a perimeter of the left upper limb larger than the right one. Suspicion of a Beckwith-Wiedemann syndrome arises, but genetic testing is not performed and the diagnosis is not confirmed. At this age the girl developed a urachal tumor, requiring surgical therapy.

The diagnosis of congenital hyperinsulinism was established at the age of three years. The suspicion was raised by the glucose intake of over $10 \mathrm{mg} / \mathrm{kg} /$ min required to control hypoglycemia during the neonatal period. In order to confirm the diagnosis, we carried out a glucagon test, which was positive. Clinically, the patient exhibited convergent strabismus and hypertrophy of the left half of the tongue, chronic constipation and incontinence. There were no clear signs of neurological distress.

The therapy consisted of enriching nutrition with fibers and Maizena corn starch, which caused the constipation and hypoglycemia to disappear. Diazoxide was also administered with a dose of $10 \mathrm{mg} / \mathrm{kg} /$ day. Initially the patient tolerated it badly, with abdominal pain and vomiting, but then developed tolerance to this medication. Under this therapy, glycemia was over $100 \mathrm{mg} / \mathrm{dl}$, which allowed us to stop administering corn starch. 
The CDKN1C was sequenced using the NGS technique, and the result was negative. It was not possible to search for the other genetic anomalies involved in the pathogeny of the Beckwith-Wiedmann syndrome. Given the child's dysmorphisms, the suspicion of a syndromic hyperinsulinism, possibly BeckwithWiedmann, still remains.

A test of oral tolerance to glucose was also performed, and it detected no kinetic anomalies in the insulin secretion (see table 1).

TABLE 1. Results of the oral glucose tolerance test

\begin{tabular}{|l|l|}
\hline Time & Glycemia value \\
\hline T0 & $50 \mathrm{mg} / \mathrm{dl}$ \\
\hline T1 $\mathrm{h}$ & $171 \mathrm{mg} / \mathrm{dl}$ \\
\hline T2h & $89 \mathrm{mg} / \mathrm{dl}$ \\
\hline
\end{tabular}

An MRI investigation with contrast identified a nodular mass located in the pancreas tail, without contrast intake, and colon dilation.

\section{DISCUSSION}

Congenital hyperinsulinism (HI) is the most frequent cause of hypoglycemia in pediatric practice, if we do not consider functional hypoglycemia.

Even though the clinical image is impressive and can sometimes have dramatic aspects, the long-term prognosis of the disease is good, as the drug-sensitive form is the only curable congenital metabolic disease.

The therapy of this disease has seen significant progress during the last years, and there are currently three pharmaceutical preparations that can be used.

There are several forms of hyperinsulinism, each of them having certain specifics regarding the treatment and the prognosis $(5,6)$.

In the authors' vision, genetic testing for this disease merits special discussion. Even though several genes involved in the pathogeny of congenital hyperinsulinism were described, no mutation was found in $50 \%$ of such patients at the time of testing. This causes genetic testing to be of little use for determining the diagnosis of congenital hyperinsulinism. The glucagon test remains much more useful in diagnosing such patients. Also very useful for the quick identification of this disease is the calculation of the glucose flow needed to control hypoglycemia. If it exceeds $10 \mathrm{mg} / \mathrm{kg} / \mathrm{min}$, the diagnosis is certain, not requiring any other tests.

It must also be mentioned that the determination of insulinemia has no real diagnostic usefulness in this disease. This is done from a peripheral vein, where the level of insulin can be falsely normal, so a normal level of insulinemia does not rule out the diagnosis of hyperinsulinism.
A particular type of $\mathrm{HI}$ is the syndromic type. In such situations, hyperinsulinemic hypoglycemia is associated to other manifestations related to that syndrome, which makes treatment more complicated, and the approach pluridisciplinary.

In this situation, the prognosis is significantly influenced by the specifics of that syndrome.

The therapy of this disease has two large components, one pharmaceutical and one surgical.

\section{Pharmaceutical therapy}

Currently, pharmaceutical therapy is the first intention, given that under the action of the recommended medication the pancreas recovers its normal function in time.

There are several drugs that can be used, which can be classified, according to the authors of this article, in three large groups: those with incontestable effectiveness, those that have sometimes proven effective but must be used with prudence, and experimental ones.

In the category with certain effectiveness we include diazoxide, octreotide and lanreotide. Diazoxide is considered to be a first line drug, and is useful for both focal forms and diffuse forms. However there are resistant forms that cannot be treated with this drug (6). Some adverse effects that are mentioned are hypertrichosis, fluid retention, thrombocytopenia, neutropenia and hyperuricemia, which indicates that careful monitoring of patients under diazoxide is mandatory (7).

Octreotide is a drug with proven effectiveness, but has several drawbacks that make it less used than diazoxide. The first one is the way of administration, which is exclusively injectable, reducing the children's therapeutic compliance. The second one consists of the adverse effects, as it may cause biliary lithiasis and necrotizing enterocolitis. An interesting aspect of this drug is that it can induce tachyphylaxis, which is a sudden loss of effectiveness. The mechanism is unknown (8).

In some carefully selected cases, we can use nifedipine or glucagon. If nifedipine is chosen, it is useful to carry out genetic testing, since it has proven to be effective in mutations of the ABCC 8 gene, but not in other forms. Nifedipine response is rare in this disease, and additional studies are needed to establish its place in the treatment of congenital HI (9).

Glucagon has been used for a long time in the treatment of congenital hyperinsulinism, especially during the acute phase and to combat hypoglycemia. Lately it has been observed that it causes rebound phenomena, which has significantly reduced its use. It 
can be used in cases of major emergency (coma), especially when intrarectal administration is not possible. Serious adverse effects are described when using it, especially for long-term use or high dosage. Such an effect is the appearance of a necrolytic migratory erythema. While rare, this can be extremely hard to correct, and careful monitoring of the patient is recommended. A real concern is also an increase in the risk of thrombosis in the case of chronic glucagon treatment. While the mechanism is unclear, there are centers that recommend adding low molecular weight heparin when choosing to use glucagon as long-term treatment (10).

To combat hypoglycemia, we prefer to use $33 \%$ glucose administered intrarectally $(1 \mathrm{ml} / \mathrm{kg})$. For the treatment of the acute phase, glucose is to be used with a flow of $16-17 \mathrm{mg} / \mathrm{kg} / \mathrm{min}$ for newborns and $12-13 \mathrm{mg} / \mathrm{kg} / \mathrm{min}$ for infants.

Two experimental drugs caught our attention, exendin and sirolimus. However, the latter is an immunosuppressor, so caution is advised (8).

Another therapy that has been tried is the ketogenic diet. Its effectiveness is questionable and there are very few reported cases of congenital $\mathrm{HI}$ that responded to this therapy (11).

\section{Surgical therapy}

Surgery is mainly targeted at focal forms. In diffuse forms, a surgical intervention is only possible in the absence of a response to pharmaceutical or dietary therapy. In order to determine the histopathological form, it is necessary to perform a PET-CT investigation, as the other imaging techniques are not recommended by experience. Unfortunately, this investigation is not possible in Romania due to bureaucratic problems, as the national PET-CT program only covers this investigation for patients with oncological illnesses and not for patients with congenital hyperinsulinism.

Several surgical techniques are used in the therapy of such patients depending on the histopathological form (focal, diffuse, atypical) (12).

In the focal form, surgery that is strictly limited to the tumor is recommended, under PET-CT guidance. This attempts to conserve as much pancreatic tissue as possible in order to avoid a secondary diabetes. In the case of deep focal forms, this technique may not be possible, requiring an extensive resection of pancreatic tissue.

In the case of diffuse forms, focal pancreatic resection is no longer possible, requiring total pancreatectomy, which will lead to secondary diabetes.

For atypical forms there is no standard technique, and the surgeon must adapt the intervention to the specifics of the case, in order to be able to save as much pancreatic tissue as possible.

In a study published by a team from the children's hospital of Philadelphia (13), of a total of 500 patients, 261 had focal forms, of which 15 were isolated insulinomas, and only 202 diffuse forms, and 37 were atypical forms. Of the patients with focal forms, 97\% were completely cured after the surgery and only 1 patient suffers from secondary diabetes. In the case of patients with diffuse forms, of a total of 202 operated patients, $47 \%$ developed secondary diabetes. For atypical forms and insulinomas, results were also satisfactory, with a curing rate $>95 \%$.

Another study published by a French team showed a rate of occurrence of secondary diabetes of $0 \%$ after surgery for focal forms, but $95 \%$ for diffuse forms (14).

A statistic published by a London group showed an incidence of $96 \%$ for diabetes after surgery for diffuse forms (15).

These statistics clearly show the importance of drug therapy and of discovering new molecules, especially for the diffuse forms. Testing response to diazoxide and octreotide is mandatory in all $\mathrm{HI}$ forms before moving to surgical therapy.

In this respect, encouraging results have been obtained using a molecule called exendin. This is an antagonist of the GLP-1 (glucagon-like-protein 1) receptors, which can be used for HI cases caused by a block in the activity of ATP-dependent potassium channels. The effectiveness of this molecule was evaluated in a placebo-controlled randomized doubleblind study carried out by a US team (16). They used a lot of 9 patients with mutations in the ABCC 8 gene and noticed that exendin treatment caused hypoglycemia to completely vanish in all these patients. These results are very encouraging, especially since mutations in the ABCC8 gene often induce resistance to diazoxide, and octreotide can cause necrotizing enterocolitis in the newborn (17).

Given that the number of patients was low, new studies are needed in connection with exendin.

\section{CONCLUSIONS}

Congenital hyperinsulinism remains to this day the only curable congenital metabolic disease. For this reason, timely diagnosis, before hyperglycemia-induced secondary brain injury, and quickly initiating the correct treatment are essential in keeping a good diagnosis.

Surgery must be delayed as much as possible, until response to drug therapy can be evaluated, especially for diffuse forms, and when used it is essential to 
perform a PET-CT investigation before surgery and use a surgical team that is experienced in this pathol- ogy in order to remove as little normal pancreatic tissue as possible and thus avoid secondary diabetes.

\section{Conflict of interest: none declared Financial support: none declared}

\section{REFERENCES}

1. Herzog RI, Sherwin RS, Rothman DL. Insulin-induced hypoglycemia and its effect on the brain: Unraveling metabolism by in vivo nuclear magnetic resonance. Diabetes. 2011;60(7):1856-8.

2. Fujita S, Rasmussen BB, Cadenas JG, Grady JJ, Volpi E. Effect of insulin on human skeletal muscle protein synthesis is modulated by insulin-induced changes in muscle blood flow and amino acid availability. Am J Physiol Endocrinol Metab. 2006 Oct;291(4):E745-54.

3. de Lonlay P, Dubois S, Valayannopoulos V, Depondt E, Ottolenghi C, Rabier D. Déficit de l'oxydation des acides gras. In Prise en charge médicale et diététique des maladies héréditaires du métabolisme. Paris: Springer, 2013, p. 307.

4. Arnoux JB, Verkarre V, Saint-Martin C, Montravers F, Brassier A, Valayannopoulos V, Brunelle F, Fournet JC, Robert JJ, Aigrain Y, Bellanné-Chantelot $\mathrm{C}$, de Lonlay $\mathrm{P}$. Congenital hyperinsulinism: Current trends in diagnosis and therapy. Orphanet J Rare Dis. 2011; 6:63.

5. Demirbilek H, Hussain K. Congenital Hyperinsulinism: Diagnosis and Treatment Update. J Clin Res Pediatr Endocrinol. 2017; 9(Suppl 2):69-87.

6. Henquin JC, Nenquin M, Sempoux C, Guiot Y, Bellanné-Chantelot $C$, Otonkoski T, de Lonlay P, Nihoul-Fékété C, Rahier J. In vitro insulin secretion by pancreatic tissue from infants with diazoxide-resistant congenital hyperinsulinism deviates from model predictions. J Clin Invest. 2011;121(10):3932-42.

7. Herrera A, Vajravelu ME, Givler S, Mitteer L, Avitabile CM, Lord K, De León DD. Prevalence of Adverse Events in Children With Congenital Hyperinsulinism Treated With Diazoxide. J Clin Endocrinol Metab. 2018;103(12):4365-4372.

8. Banerjee I, Salomon-Estebanez M, Shah P, Nicholson J, Cosgrove KE, Dunne MJ. Therapies and outcomes of congenital hyperinsulinisminduced hypoglycaemia. Diabet Med. 2019;36(1):9-21.

9. Khawash P, Hussain K, Flanagan SE, Chatterjee S, Basak D. Nifedipine in Congenital Hyperinsulinism - A Case Report. J Clin Res Pediatr Endocrinol. 2015;7(2):151-4.
10. Worth C, Yau D, Salomon Estebanez M, O'Shea E, Cosgrove K, Dunne M, Banerjee I. Complexities in the medical management of hypoglycaemia due to congenital hyperinsulinism. Clinical Endocrinology 2020;92(5):387-395.

11. Maiorana A, Manganozzi L, Barbetti F, Bernabei S, Gallo G, Cusmai R, Caviglia S, Dionisi-Vici C. Ketogenic diet in a patient with congenital hyperinsulinism: A novel approach to prevent brain damage. Orphanet Journal of Rare Diseases 2015;10(1):120.

12. Barthlen W, Mohnike W, Mohnike K. Techniques in pediatric surgery: Congenital hyperinsulinism. Horm Res Paediatr. 2011;75(4):304-10.

13. Adzick NS, De Leon DD, States LJ, Lord K, Bhatti TR, Becker SA, Stanley CA. Surgical treatment of congenital hyperinsulinism: Results from 500 pancreatectomies in neonates and children. J Pediatr Surg. 2019;54(1):27-32.

14. Beltrand J, Caquard M, Arnoux JB, Laborde K, Velho G, Verkarre V, Rahier J, Brunelle F, Nihoul-Fékété C, Saudubray JM, Robert JJ, de Lonlay P. Glucose metabolism in 105 children and adolescents after pancreatectomy for congenital hyperinsulinism. Diabetes Care. 2012; 35(2):198-203.

15. Arya VB, Senniappan S, Demirbilek H, Alam S, Flanagan SE, Ellard $\mathrm{S}$, Hussain K. Pancreatic endocrine and exocrine function in children following near-total pancreatectomy for diffuse congenital hyperinsulinism. PLoS One. 2014;9(5):e98054.

16. Calabria AC, Li C, Gallagher PR, Stanley CA, De León DD. GLP-1 receptor antagonist exendin-(9-39) elevates fasting blood glucose levels in congenital hyperinsulinism owing to inactivating mutations in the ATP-sensitive K+ channel. Diabetes. 2012;61(10):2585-91.

17. McMahon AW, Wharton GT, Thornton P, De Leon DD. Octreotide use and safety in infants with hyperinsulinism. Pharmacoepidemiol Drug Saf. 2017;26(1):26-31. 